\title{
Development of a competing risk nomogram for the prediction of cause-specific mortality in patients with thymoma: a population- based analysis
}

\author{
Tao Zhang ${ }^{1}$, Lipin Liu ${ }^{2}$, Bin Qiu ${ }^{3}$ \\ ${ }^{1}$ Department of Radiation Oncology, National Cancer Center/National Clinical Research Center for Cancer/Cancer Hospital, Chinese Academy \\ of Medical Science and Peking Union Medical College, Beijing, China; ${ }^{2}$ Department of Radiation Oncology, Beijing Hospital, National Center of \\ Gerontology, Institute of Geriatric Medicine, Chinese Academy of Medical Sciences, Beijing, China; ${ }^{3}$ Department of Thoracic Surgery, National \\ Cancer Center/National Clinical Research Center for Cancer/Cancer Hospital, Chinese Academy of Medical Science and Peking Union Medical \\ College, Beijing, China \\ Contributions: (I) Conception and design: T Zhang, B Qiu; (II) Administrative support: T Zhang; (III) Provision of study materials or patients: T \\ Zhang, L Liu; (IV) Collection and assembly of data: All authors; (V) Data analysis and interpretation: T Zhang, B Qiu; (VI) Manuscript writing: All \\ authors; (VII) Final approval of manuscript: All authors. \\ Correspondence to: Bin Qiu. Department of Thoracic Surgery, National Cancer Center/National Clinical Research Center for Cancer/Cancer \\ Hospital, Chinese Academy of Medical Science and Peking Union Medical College, No. 17 Panjiayuan Nanli, Chaoyang District, Beijing, China. \\ Email: drqiubin@aliyun.com.
}

Background: This study was developed to assess the odds of cause-specific mortality and other types of mortality in thymoma patients. In addition, these analyses were leveraged to develop a comprehensive competing risk model-based nomogram capable of predicting cause-specific mortality as a result of thymoma. Methods: Thymoma patients included within the Surveillance, Epidemiology, and End Results (SEER) database from 2004-2016 were identified, and the odds of cause-specific mortality due to thymoma and other forms of mortality for these patients were estimated. In addition, Fine and Gray's proportional subdistribution hazard model was constructed, and a competing risk nomogram was developed using this model that was capable of predicting the odds of 3-, 5-, and 10-year cause-specific mortality in thymoma patients.

Results: In total, 1,591 relevant cases in the SEER database were selected for analysis. In this patient cohort, the respective 5 -year cumulative incidence rates for cause-specific mortality and mortality attributable to other causes were $12.4 \%$ and $8.2 \%$. Variables significantly associated with cause-specific mortality included age, chemotherapy, surgery, and Masaoka stage. Additionally, the odds of other-causespecific mortality rose with increasing patient age, and chemotherapy was correlated with other-causespecific mortality. The competing risk nomogram that was developed exhibited good discriminative ability as a means of predicting cause-specific mortality, as evidenced by a concordance index (C-index) value of 0.84 . Calibration curves further revealed excellent consistency between predicted and actual mortality when using this nomogram.

Conclusions: In summary, we herein assessed the odds of cause-specific and other-cause-specific mortality among thymoma patients, and we designed a novel nomogram capable of predicting cause-specific mortality for thymoma, providing a promising tool that may be of value in the context of individualized patient prognostic evaluation.

Keywords: Thymoma; competing risk analysis; cause-specific mortality; cumulative incidence; nomogram

Submitted Jun 04, 2021. Accepted for publication Oct 09, 2021.

doi: $10.21037 /$ jtd-21-931

View this article at: https://dx.doi.org/10.21037/jtd-21-931 


\section{Introduction}

Thymoma is a relatively rare malignancy of thymic epithelial cells accounting for $0.2-1.5 \%$ of all malignancies (1). However, thymoma represents approximately $50 \%$ of all malignancies arising from anterior mediastinum (2). With relatively indolent behavior, thymoma presents substantial heterogeneity in prognosis. The most widely used staging systems for thymoma are Masaoka staging system and American Joint Commission on Cancer (AJCC) 8th Edition of TNM classification. The Masaoka staging system relies primarily on primary tumor extension and the degree of involvement beyond the thymus (3). The recently proposed AJCC 8th Edition of TNM classification is based on combination of primary tumor local invasion, nodal involvement and metastatic spread (4). However, both the Masaoka system and the TNM staging system are inadequate to explain the large heterogeneity in outcomes for patients with thymoma. Recent studies have reported that age, completeness of resection and histologic type were important prognostic factors supplementing stage (5-7). Therefore, the development of a technically feasible and clinically accessible predictive tool that incorporates patientrelated, tumor-related, and treatment-related prognostic factors is needed.

With regard to age distribution, the incidence of thymoma increases with age into the eighth decade of life and then decreases (8). The mean age at diagnosis is 50-60 years old (2). Besides, thymoma is characterized by its indolent behavior and long natural history with 5-year overall survival of approximately $90 \%$ (8-10). As such, longterm survivors and elderly individuals often die of causes not specific to this particular form of malignancy. It is thus important to take such other causes of mortality into account when predicting thymoma patient prognosis. While several studies have assessed thymoma patient outcomes, a majority of these analyses have employed the Kaplan-Meier analytical approach, which is likely not appropriate given that it treats competing events as independent censoring, thereby potentially leading to an overestimation of cancerspecific death. A competing risk model is better suited to this analytical context, as it takes the type of censoring into consideration, thereby allowing for more reliable discrimination between the effects of particular risk factors on different outcome events (11).

Several recent studies have described the development of competing risk nomograms for the evaluation of patients with thyroid, breast, and renal cancer (12-14), but no such nomograms have been developed to conduct a competing risk analysis of thymoma. This study selected all cases with thymoma registered in the Surveillance, Epidemiology, and End Results (SEER) database between 2004 and 2016 as well as performed a competing-risk analysis. We evaluated the probabilities for cause-specific mortality and other causes of mortality, and constructed a competing risk nomogram to predict cause-specific mortality for thymoma.

We present the following article in accordance with the TRIPOD reporting checklist (available at https://dx.doi. org/10.21037/jtd-21-931).

\section{Methods}

\section{Patients and data collection}

For the present study, patients in the SEER 18 registry maintained by the National Cancer Institute (1975-2016; dataset submitted November 2018) were analyzed, with thymoma patients being extracted with the SEER*Stat software (v 8.3.9). All patients diagnosed with histologically confirmed thymoma from 2004-2016 were included in this analysis. No Institutional Review Board approval for this study was required owing to the anonymized and openaccess nature of the SEER database.

The International Classification of Diseases for Oncology, third edition (ICD-O-3) criteria were used to classify tumor site and histology. Patients enrolled in this study were those with the ICD-O-3 site code C37.9 (thymus) and the ICD-O-3 histology codes 8580-8585. Patients were excluded if they met the following criteria: (I) patients $<18$ years old at the time of diagnosis; (II) patients diagnosed upon autopsy or by death certificate; (III) patients with other primary malignancies; (IV) patients for whom complete data pertaining to age at diagnosis, sex, ethnicity, stage, treatment, histology, vital status or survival month, or SEER cause of death records were not available; (V) patients with a recorded survival duration of $<1$ month. Variables for analysis were race, age, sex, histology, stage, surgery, radiotherapy and chemotherapy. Staging in the SEER database was grouped into the localized, regional, and distant disease categories, and we regrouped included thymoma patients into three groups based on corresponding Masaoka stages as follows: stage I/IIA (localized disease), stage IIB (regional disease), and stage III/IV (distant disease or any positive lymph nodes). The age at diagnosis was divided into four groups: $<50,50-59,60-69$ and $\geq 70$ years. The histology was divided into four groups: A/AB, B1/B2, 
B3 and not otherwise specified (NOS).

\section{Statistical analysis}

Cause-specific and other-cause-specific mortality were treated as competing events in this study, with the probability of death being evaluated using a cumulative incidence function (CIF). Differences in cumulative incidence between groups were compared via Gray's test (15), and the combined effects of patient- and treatment-related characteristics on cause-specific and other-cause-specific mortality were assessed with a proportional subdistribution hazard model (16). Fine and Gray's model was used to develop a competing risk nomogram in order to predict cancer-specific mortality in thymoma patients (17). Concordance index (C-index) values and calibration plots were used to assess the performance of this nomogram. Calibration plots were generated via a bootstrap cross-validation approach with $1,000 \times$ resampling. $\mathrm{R}$ version 3.6.3 software (http://www.r-project.org/) was used for all statistical analyses. The 'rms', 'cmprsk', and 'mstate' R packages were used for model and nomogram construction, while model performance was assessed using the 'pec' package. A two-sided $\mathrm{P}<0.05$ was the threshold of significance.

\section{Results}

We identified 1,591 patients with histologically confirmed thymoma between 2004 and 2016 from SEER database. Patients' characteristics are shown in Table 1. The median age of these patients at diagnosis was 58 years, with $28.9 \%$, $23.7 \%, 26.5 \%$, and $20.9 \%$ of patients being $<50,50-59$, $60-69$, and $\geq 70$ years old, respectively. Of the 1,591 patients, $50.3 \%$ were female. The majority of patients were white $(66.6 \%)$. The proportions of patients with WHO histological classification $\mathrm{A} / \mathrm{AB}, \mathrm{B} 1 / \mathrm{B} 2, \mathrm{~B} 3$ and NOS were $26.7 \%, 28.9 \%, 17.9 \%$ and $26.5 \%$, respectively. With respect to tumor stage distribution, $35.3 \%, 41.3 \%$ and $23.4 \%$ of patients presented with Masaoka stage I-IIA, IIB, and IIIIV, respectively. A majority of patients $(82.8 \%)$ underwent surgical treatment, while $45.7 \%$ and $28.6 \%$ of patients underwent radiotherapy and chemotherapy, respectively.

The median follow-up duration for patients included in this study was 65 months (interquartile range, 28106 months). Up to the last follow up, a total of 1,241 patients $(78.0 \%)$ had been censored, and 350 patients (22.0\%) had died during follow up, including 195 deaths
(12.3\%) from thymoma and 155 (9.7\%) from other causes. The respective 5 -year cumulative incidence rates for cause-specific mortality and other-cause-specific mortality were $12.4 \%$ and $8.2 \%$ (Table 1 ). In a competing risk analysis, older age, B3 histology, advanced stage, no surgical treatment, and chemotherapy treatment were significantly associated with cause-specific mortality $(\mathrm{P}<0.001)$. In addition, older age, no surgical treatment, and chemotherapy were significantly associated with a higher risk of other-cause-specific mortality. Corresponding CIF curves based upon these variables are shown in Figure 1.

Proportional subdistribution hazard models were utilized to assess the odds of cause-specific thymoma patient mortality (Table 2). In this analysis, age, surgical treatment, chemotherapy, and Masaoka stage were found to independently predict cause-specific patient mortality. Compared with Masaoka stage I/IIA disease, patients with stage IIB disease [subdistribution hazard ratio $(\mathrm{SHR})=1.95$, 95\% confidence interval (CI), 1.15-3.28, $\mathrm{P}=0.013]$ and stage III/IV disease (SHR =3.18, 95\% CI, 1.87-5.40, $\mathrm{P}<0.001$ ) had higher risk of cause-specific death. Patients who did not receive surgery $(\mathrm{SHR}=3.88,95 \% \mathrm{CI}, 2.74-5.48$, $\mathrm{P}<0.001)$ experienced increased probability of mortality from thymoma. Decreased probability of cause-specific mortality was observed in patients without chemotherapy than those who underwent chemotherapy $(\mathrm{SHR}=0.41$, 95\% CI, 0.29-0.59, $\mathrm{P}<0.001)$. After adjustment for various factors, histology was no longer predictive of cause-specific mortality. Age was significantly predictive of both cause specific death and other causes of death; with older patients carrying a higher risk of mortality. Besides, a higher cumulative incidence of other causes of mortality was observed in patients without chemotherapy $(\mathrm{SHR}=2.13$, 95\% CI, 1.30-3.49, $\mathrm{P}=0.003$ ).

Fine and Gray's model was used to develop a predictive nomogram capable of gauging the odds of 3-, 5-, and 10 -year thymoma patient cause-specific survival based upon the sum of points corresponding to the included characteristics (Figure 2). This model exhibited good discriminative ability, with a C-index value of 0.84 . Calibration plots exhibited good consistency between actual and predicted probability of cause-specific mortality in these patients (Figure 3).

\section{Discussion}

Herein, we conducted an analysis of mortality rates among thymoma patients recorded in the SEER database from 
Table 1 Five-year cumulative incidences of mortality among patients with thymoma

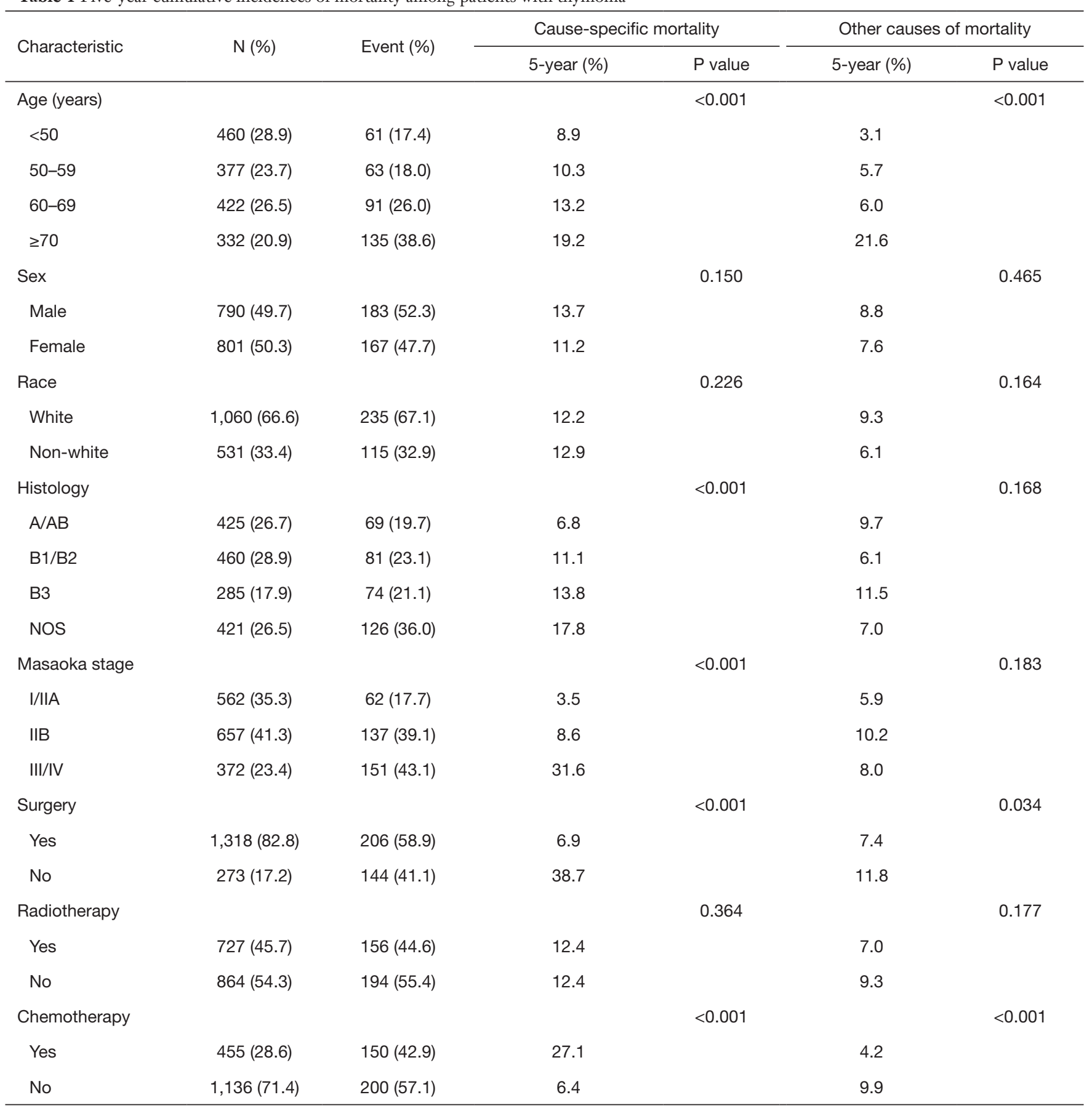

NOS, not otherwise specified.

2004-2016. In this patient population, the respective 5 -year cumulative incidence rates of cause-specific and other-cause-specific mortality were $12.4 \%$ and $8.2 \%$. This study is the first to our knowledge to have explored the cumulative incidence of cause-specific mortality associated with thymoma and to develop a competing risk nomogram model capable of predicting such mortality.

Identification of prognostic factors is critical in optimizing treatment for patients with cancer. The well recognized prognostic factors for thymoma include tumor 

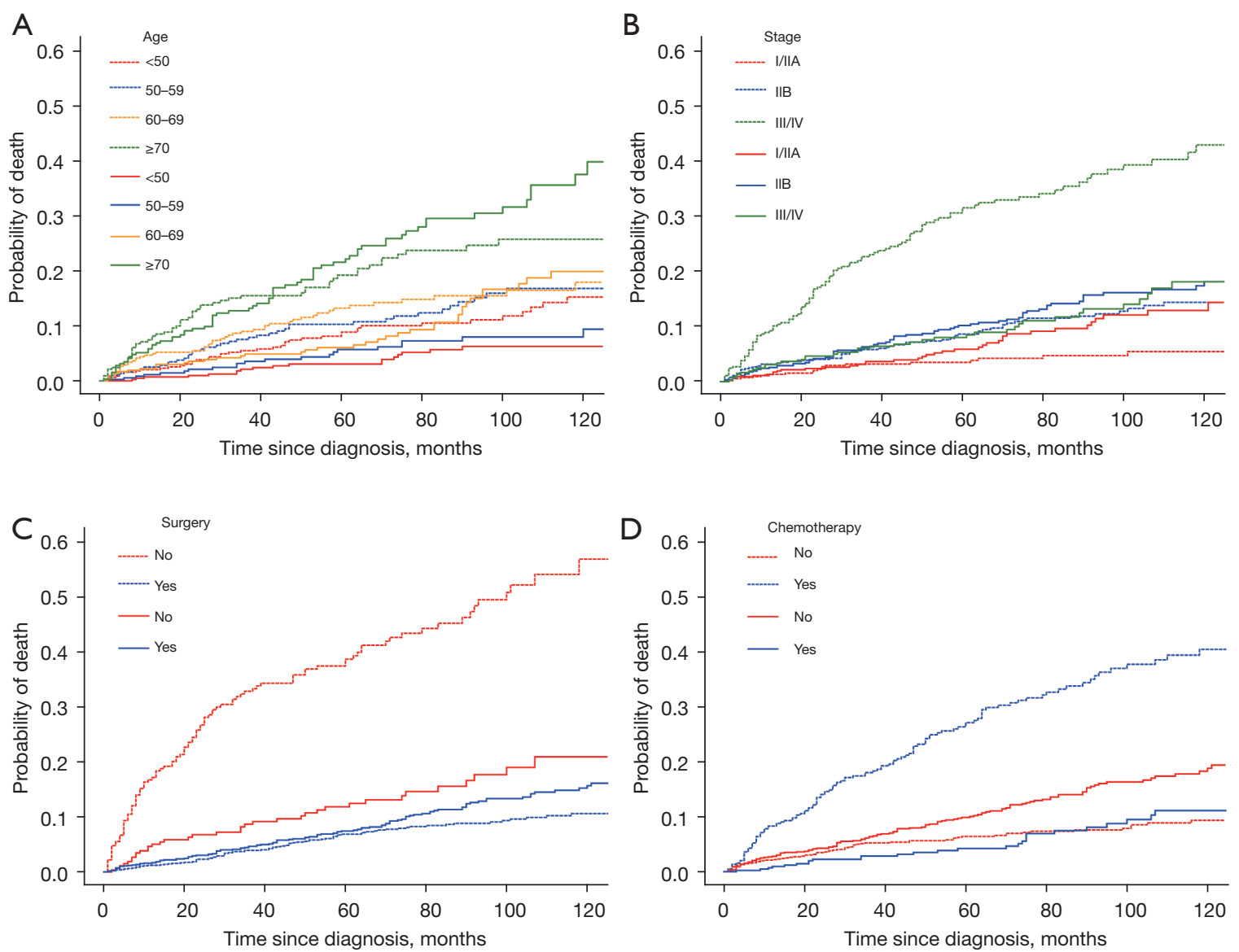

Figure 1 Cumulative cause-specific and other-cause-specific mortality curves stratified according to patient (A) age; (B) Masaoka stage; (C) surgery; (D) chemotherapy. Cause-specific mortality is represented by a dotted line, while all other causes of mortality are represented by a solid line.

stage and resection status $(6,7,18)$, which is consistent with our results that advanced stage predicted unfavorable causespecific survival. Resection status has been considered as an important favorable prognostic factor and should be classified as complete resection (R0), microscopically incomplete resection (R1) and grossly incomplete resection (R2). Patients with R0 resection could have significantly superior survival than those with R1 or R2 resection $(6,18)$. However, data regarding resection status was incomplete in SEER database.

Another potential prognostic factor is patient age. Rea et al. demonstrated that age had no effect on survival after adjusting for multivariates (19). Pediatric thymoma has been reported to present a more aggressive behavior and be associated with an increased risk of distant metastasis resulting in unfavorable survival compared with adults (20). However, our study excluded pediatric thymoma and demonstrated that the risk of both cause-specific mortality and other causes of mortality increased with age, which is consistent with studies by Filosso and Ruffini $(6,21)$. Elder patients are associated with more comorbidities and poorer performance status which hinder them from receiving radical or aggressive treatment. The decrease in therapeutic intensity and comorbidities-related non-cancer death may result in the inferior prognosis in elderly.

According to the WHO histological classification, thymoma comprises a spectrum of unique tumors subdivided into type $A, A B, B 1, B 2$, and B3. The independent prognostic value of histologic type for thymoma is controversial. The worldwide retrospective study by International Thymic Malignancy Interest Group (ITMIG) reported that WHO histologic type seemed to correlate well with Masaoka stage. The majority of type $\mathrm{A}$ and $\mathrm{AB}$ tumors were Masaoka stage I or II, while the $\mathrm{B}$ 
Table 2 Proportional subdistribution hazard models of probabilities of mortality for patients with thymoma

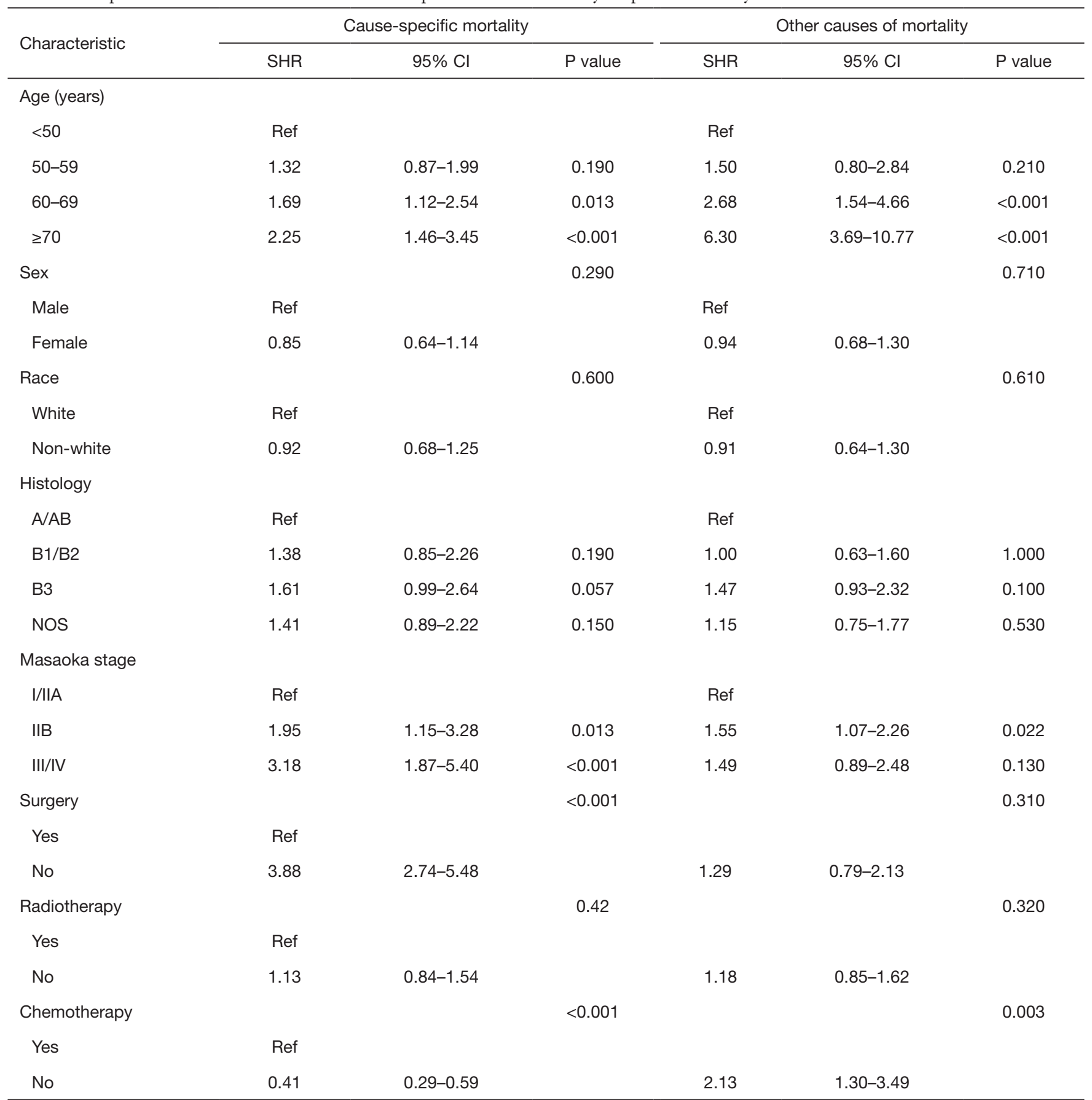

SHR, subdistribution hazard ratio; $\mathrm{Cl}$, confidence interval; NOS, not otherwise specified.

subtypes especially B3 subtype tended to present the higher Masaoka stages (7). Studies by Rea and Okumura suggested histologic type had independent prognostic value $(19,22)$. Nevertheless, Weis et al. (7) suggested that the effect of histologic type on recurrence was only observed in stage $\mathrm{I} /$ II thymoma, in type $\mathrm{A}$ and $\mathrm{AB}$ versus $\mathrm{B} 1$ to $\mathrm{B} 3$ thymoma. $\mathrm{A}$ multivariate study of the large database by European Society of Thoracic Surgeons (6) found no independent correlation 


Points
Age, years
Sex
Race
Histology
Stage
Surgery
Radiotherapy
Chemotherapy
Total Points
3-year probability of CSM
5-year probability of CSM
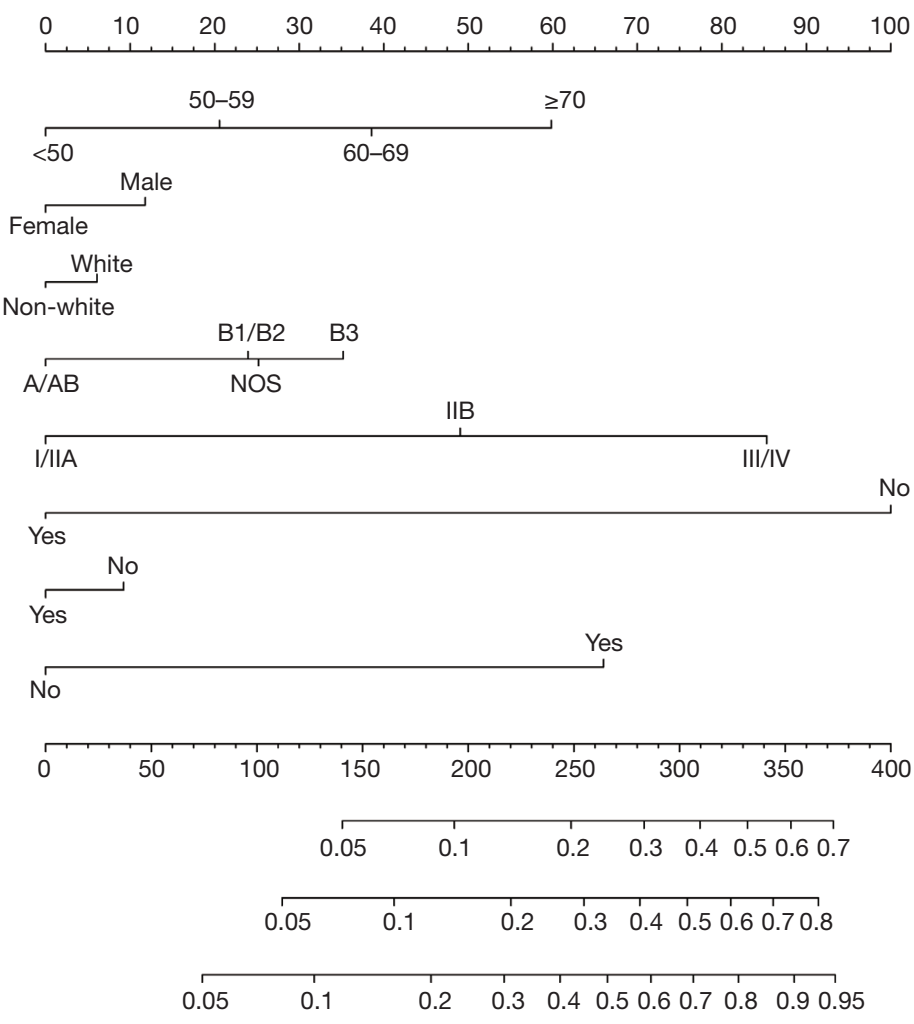

Figure 2 Competing risk nomogram for the prediction of 3-, 5-, 10-year cause-specific mortality associated with thymoma. CSM, causespecific mortality.

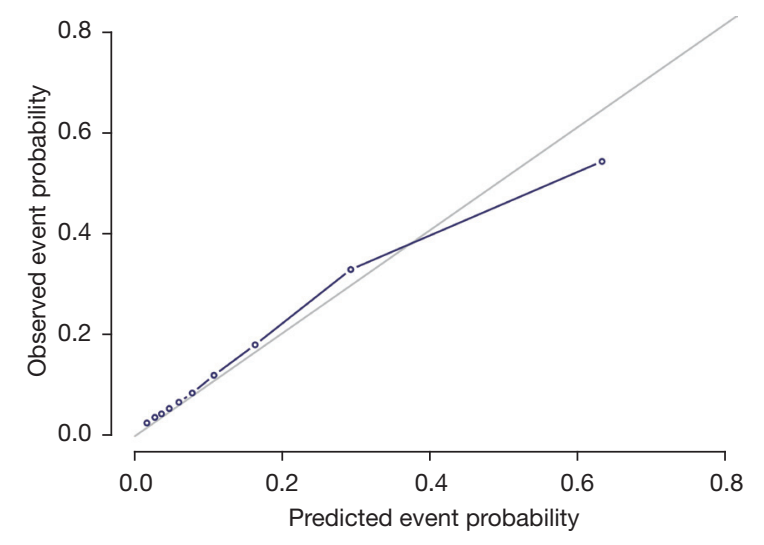

Figure 3 Calibration plot for cause-specific mortality nomogram in thymoma. The $\mathrm{x}$-axis and $\mathrm{y}$-axis respectively correspond to the predicted odds of cause-specific survival and the actual observed incidence of cause-specific survival.

between histologic type and survival or recurrence, which is consistent with our results. In our study, histology was correlated with cause-specific mortality in univariate analysis while failed to maintain its independent prognostic role after adjusting for various multivariates. In conclusion, the independent prognostic role of histologic type for thymoma has not been well defined.

Treatment modalities for thymoma include surgery, radiotherapy, chemotherapy and targeted therapy. Given the rarity of thymoma, the optimal utilization and sequencing of these treatment modalities have not been well defined through large randomized prospective studies. To date, complete surgical resection remains the mainstay treatment for thymoma. Complete resection is also a significant favorable prognostic factor. However, for locally advanced disease, complete tumor resection is often difficult to achieve. Research on the beneficial role of subtotal resection on survival for unresectable thymoma has shown inconsistent results. Some studies have reported better survival with debulking surgery combined with adjuvant radiotherapy $(23,24)$ while others demonstrated no benefit from debulking surgery over biopsy alone $(25,26)$. In 2015 , a meta-analysis of retrospective studies demonstrated that debulking surgery for unresectable thymoma may 
improve overall survival and be considered as an effective local treatment method for unresectable thymoma (27). However, the meta-analysis was limited by the existence of large heterogeneity between the included studies. In our study, surgery was associated with improved cause-specific survival, which is accordant with previous studies suggesting surgery as the cornerstone of a multimodal therapy for thymoma.

The role of radiotherapy in the management of thymoma remains somewhat controversial. For unresectable disease, definitive radiotherapy is recommended. To date, no randomized studies exist evaluating the role of adjuvant radiotherapy. Multiple retrospective studies have suggested that radiotherapy reduced recurrence rates and improved outcomes for incompletely resected thymoma (28-30). Adjuvant radiotherapy is not recommended for completely resected stage I thymoma (31). The role of adjuvant radiotherapy for completely resected stage II-III thymoma is debatable based on mixed results from several large-size dataset retrospective studies (30,32-34). Most retrospective data were subject to heterogeneity in resection status and histology type between radiotherapy and nonradiotherapy groups. The study by ITMIG represented the largest dataset focusing on completely resected stage II-III thymoma and demonstrated a beneficial role of adjuvant radiotherapy in this subgroup (34). However, radiotherapy was not associated with cause-specific survival in our study. Thymoma tumors are sensitive to chemotherapeutic treatment, with respective $1 / 3$ and $2 / 3$ complete and partial response rates. We found chemotherapy treatment to be a negative prognostic factor in thymoma patients. However, caution is warranted when interpreting this result owing to the potential for selection bias given that patients selected to undergo chemotherapy are more likely to have advanced staging, locoregional recurrence, or distant metastases.

Considering the increased incidence of thymoma with age and long-term survival rates for patients, competing non-cancer mortality represents a critical consideration when predicting prognosis for decision making. With the risk of competing other causes of mortality increases, and the benefit of increasing treatment intensity potentially diminishes. For the first time, we built a competing risk nomogram to predict cause-specific death for thymoma. This study has several strengths. The data are derived from a population-based cohort with relatively large sample size. Despite the rarity of thymoma, the SEER database provided a sufficiently large sample size to develop and validate the competing risk nomogram. In addition, the nomogram has several clinical implications, such as helping clinicians improve outcome prediction and tailor individualized treatment. By combining a few predictors, the nomogram demonstrated good predictive ability and was also simple to use.

This study has several limitations that must be acknowledged. Data regarding some important prognostic factors such as margin status, tumor size and specific Masaoka stage were not available in SEER database. Masaoka stage was not specifically recorded, but was assigned based on recorded information instead. In addition, comorbidity score was reported to be significantly associated with other causes of mortality in other cancer types $(35,36)$. However, this variable was not included in SEER database. Lastly, while internal bootstrapping-based validation was conducted to evaluate the performance of this nomogram and it performed well, external validation using a separate patient population will be essential to establish the relative accuracy of this model.

\section{Conclusions}

In this analysis, the SEER database was leveraged to assess cause-specific and other-cause-specific mortality among thymoma patients. We additionally constructed a novel predictive nomogram capable of estimating 3-, 5-, and 10 -year cause-specific mortality among thymoma patients. This nomogram exhibited relatively good performance, and may thus offer value as a reliable tool for individual prognostic analyses of thymoma patients. However, additional external validation of this model remains to be conducted.

\section{Acknowledgments}

Funding: None.

\section{Footnote}

Reporting Checklist: The authors have completed the TRIPOD reporting checklist. Available at https://dx.doi. org/10.21037/jtd-21-931

Peer Review File: Available at https://dx.doi.org/10.21037/ jtd-21-931

Conflicts of Interest: All authors have completed the ICMJE uniform disclosure form (available at https://dx.doi. 
org/10.21037/jtd-21-931). The authors have no conflicts of interest to declare.

Ethical Statement: The authors are accountable for all aspects of the work in ensuring that questions related to the accuracy or integrity of any part of the work are appropriately investigated and resolved. The study was conducted in accordance with the Declaration of Helsinki (as revised in 2013).

Open Access Statement: This is an Open Access article distributed in accordance with the Creative Commons Attribution-NonCommercial-NoDerivs 4.0 International License (CC BY-NC-ND 4.0), which permits the noncommercial replication and distribution of the article with the strict proviso that no changes or edits are made and the original work is properly cited (including links to both the formal publication through the relevant DOI and the license). See: https://creativecommons.org/licenses/by-nc-nd/4.0/.

\section{References}

1. Engels EA. Epidemiology of thymoma and associated malignancies. J Thorac Oncol 2010;5:S260-5.

2. Drevet G, Collaud S, Tronc F, et al. Optimal management of thymic malignancies: current perspectives. Cancer Manag Res 2019;11:6803-14.

3. Koga K, Matsuno Y, Noguchi M, et al. A review of 79 thymomas: modification of staging system and reappraisal of conventional division into invasive and non-invasive thymoma. Pathol Int 1994;44:359-67.

4. Detterbeck FC, Stratton K, Giroux D, et al. The IASLC/ ITMIG Thymic Epithelial Tumors Staging Project: proposal for an evidence-based stage classification system for the forthcoming (8th) edition of the TNM classification of malignant tumors. J Thorac Oncol 2014;9:S65-72.

5. Li JF, Hui BG, Li X, et al. Video-assisted thoracic surgery for thymoma: long-term follow-up results and prognostic factors-single-center experience of 150 cases. J Thorac Dis 2018;10:291-7.

6. Ruffini E, Detterbeck F, Van Raemdonck D, et al. Tumours of the thymus: a cohort study of prognostic factors from the European Society of Thoracic Surgeons database. Eur J Cardiothorac Surg 2014;46:361-8.

7. Weis CA, Yao X, Deng Y, et al. The impact of thymoma histotype on prognosis in a worldwide database. J Thorac Oncol 2015;10:367-72.

8. Engels EA, Pfeiffer RM. Malignant thymoma in the
United States: demographic patterns in incidence and associations with subsequent malignancies. Int $\mathrm{J}$ Cancer 2003;105:546-51.

9. Merveilleux du Vignaux C, Dansin E, Mhanna L, et al. Systemic Therapy in Advanced Thymic Epithelial Tumors: Insights from the RYTHMIC Prospective Cohort. J Thorac Oncol 2018;13:1762-70.

10. Zhao Y, Shi J, Fan L, et al. Surgical treatment of thymoma: an 11-year experience with 761 patients. Eur J Cardiothorac Surg 2016;49:1144-9.

11. Carmona R, Zakeri K, Green G, et al. Improved Method to Stratify Elderly Patients With Cancer at Risk for Competing Events. J Clin Oncol 2016;34:1270-7.

12. Hanrahan EO, Gonzalez-Angulo AM, Giordano SH, et al. Overall survival and cause-specific mortality of patients with stage T1a,bN0M0 breast carcinoma. J Clin Oncol 2007;25:4952-60.

13. Kutikov A, Egleston BL, Wong YN, et al. Evaluating overall survival and competing risks of death in patients with localized renal cell carcinoma using a comprehensive nomogram. J Clin Oncol 2010;28:311-7.

14. Yang L, Shen W, Sakamoto N. Population-based study evaluating and predicting the probability of death resulting from thyroid cancer and other causes among patients with thyroid cancer. J Clin Oncol 2013;31:468-74.

15. Gray RJ. A class of k-sample tests for comparing the cumulative incidence of a competing risk. Ann Stat 1988;16:1141-54.

16. Fine JP, Gray RJ. A proportional hazards model for the subdistribution of competing risks in survival analysis. J Am Stat Assoc 1999;94:496-509.

17. Zhang Z, Geskus RB, Kattan MW, et al. Nomogram for survival analysis in the presence of competing risks. Ann Transl Med 2017;5:403.

18. Detterbeck F, Youssef S, Ruffini E, et al. A review of prognostic factors in thymic malignancies. J Thorac Oncol 2011;6:S1698-704.

19. Rea F, Marulli G, Girardi R, et al. Long-term survival and prognostic factors in thymic epithelial tumours. Eur J Cardiothorac Surg 2004;26:412-8.

20. Liu M, Wang C, Gao L, et al. Clinical significance of age at diagnosis among patients with thymic epithelial tumors: a population-based study. Aging (Albany NY) 2020;12:4815-21.

21. Filosso PL, Evangelista A, Ruffini E, et al. Does myasthenia gravis influence overall survival and cumulative incidence of recurrence in thymoma patients? A Retrospective clinicopathological multicentre analysis on 
797 patients. Lung Cancer 2015;88:338-43.

22. Okumura M, Ohta M, Tateyama H, et al. The World Health Organization histologic classification system reflects the oncologic behavior of thymoma: a clinical study of 273 patients. Cancer 2002;94:624-32.

23. Kondo K, Monden Y. Therapy for thymic epithelial tumors: a clinical study of 1,320 patients from Japan. Ann Thorac Surg 2003;76:878-84; discussion 884-5.

24. Maggi G, Casadio C, Cavallo A, et al. Thymoma: results of 241 operated cases. Ann Thorac Surg 1991;51:152-6.

25. Cohen DJ, Ronnigen LD, Graeber GM, et al. Management of patients with malignant thymoma. J Thorac Cardiovasc Surg 1984;87:301-7.

26. Fan C, Ge H, Zhang S, et al. Impact of Definitive Radiotherapy and Surgical Debulking on Treatment Outcome and Prognosis for Locally Advanced MasaokaKoga stage III Thymoma. Sci Rep 2020;10:1735.

27. Hamaji M, Kojima F, Omasa M, et al. A meta-analysis of debulking surgery versus surgical biopsy for unresectable thymoma. Eur J Cardiothorac Surg 2015;47:602-7.

28. Basse C, Thureau S, Bota S, et al. Multidisciplinary Tumor Board Decision Making for Postoperative Radiotherapy in Thymic Epithelial Tumors: Insights from the RYTHMIC Prospective Cohort. J Thorac Oncol 2017;12:1715-22.

29. Forquer JA, Rong N, Fakiris AJ, et al. Postoperative radiotherapy after surgical resection of thymoma: differing roles in localized and regional disease. Int J Radiat Oncol Biol Phys 2010;76:440-5.

30. Jackson MW, Palma DA, Camidge DR, et al. The Impact of Postoperative Radiotherapy for Thymoma and Thymic Carcinoma. J Thorac Oncol 2017;12:734-44.

31. Zhang H, Lu N, Wang M, et al. Postoperative radiotherapy for stage I thymoma: a prospective randomized trial in 29 cases. Chin Med J (Engl) 1999;112:136-8.

32. Liu Q, Gu Z, Yang F, et al. The role of postoperative radiotherapy for stage I/II/III thymic tumor-results of the ChART retrospective database. J Thorac Dis 2016;8:687-95.

33. Omasa M, Date H, Sozu T, et al. Postoperative radiotherapy is effective for thymic carcinoma but not for thymoma in stage II and III thymic epithelial tumors: the Japanese Association for Research on the Thymus Database Study. Cancer 2015;121:1008-16.

34. Rimner A, Yao X, Huang J, et al. Postoperative Radiation Therapy Is Associated with Longer Overall Survival in Completely Resected Stage II and III ThymomaAn Analysis of the International Thymic Malignancies Interest Group Retrospective Database. J Thorac Oncol 2016;11:1785-92.

35. Bøje CR, Dalton SO, Grønborg TK, et al. The impact of comorbidity on outcome in 12623 Danish head and neck cancer patients: a population based study from the DAHANCA database. Acta Oncol 2013;52:285-93.

36. Kim YH, Roh JL, Kim SB, et al. Risk factors for competing non-cancer mortality after definitive treatment for advanced-stage head and neck cancer. Oral Dis 2018;24:1217-25.
Cite this article as: Zhang T, Liu L, Qiu B. Development of a competing risk nomogram for the prediction of causespecific mortality in patients with thymoma: a population-based analysis. J Thorac Dis 2021;13(12):6838-6847. doi: 10.21037/jtd21-931 